INVESTIGACIÓN

http://doi.org/10.15198/seeci.2020.53.23-35

Recibido: 11/06/2020 --- Aceptado: 28/08/2020 --- Publicado: 15/11/2020

\title{
COMUNICACIÓN EFECTIVA Y DOMINIO AFECTIVO EN EL APRENDIZAJE DE LAS MATEMÁTICAS
}

\section{EFFECTIVE COMMUNICATION AND AFFECTIVE DOMAIN IN THE LEARNING OF MATHEMATICS}

Mercedes de la Oliva Fernández ${ }^{1}$ : Consultor independiente. España. mdeoliva@gmail.com

\section{RESUMEN}

La matemática ha sido y seguirá siendo fundamento riguroso e ineludible en una gran cantidad de disciplinas y especialidades, lo que muestra la necesidad de su inclusión y su potencial en muchas propuestas curriculares dentro de la formación académica a todos los niveles. Sin embargo, diversos autores la refieren como la menos popular de las disciplinas en casi cualquier plan de estudios. Esta falta de popularidad $y$, en consecuencia, las dificultades que se presentan tanto en su aprendizaje como en su enseñanza, tienen múltiples razones. Lo cierto es que está presente una significativa aversión de los estudiantes hacia las matemáticas, generando así los bajos niveles de rendimiento en esta área del conocimiento. En el presente artículo, se expondrán dos elementos considerados determinantes para el aprendizaje de las matemáticas. En primer lugar, la comunicación efectiva y el lenguaje como foco para que profesores y estudiantes lleguen a acuerdos sobre los significados de los objetos matemáticos compartidos $y$, en segundo lugar, el dominio afectivo, relacionado con las creencias, las actitudes y las emociones, que determinan la valoración subjetiva que cada individuo demuestra cuando se enfrenta a procesos de aprendizaje relacionados con las matemáticas y que son, precisamente, los responsables de las reacciones, más negativas que positivas, que expresan.

PALABRAS CLAVE: Comunicación efectiva - dominio afectivo - aprendizaje matemáticas.

\section{ABSTRACT}

Mathematics has been and will continue to be a rigorous and unavoidable foundation in a large number of disciplines and specialties, which shows the need for its

\footnotetext{
${ }^{1}$ Mercedes de la Oliva Fernández. Doctora en didáctica, organización escolar y didácticas especiales por la UNED, con amplia experiencia en el aprendizaje y la enseñanza de las matemáticas universitarias. mdeoliva@gmail.com
} 
inclusion and its potential in many curricular proposals within academic training at all levels. However, various authors refer to it as the least popular of the disciplines in almost any curriculum. This lack of popularity and, consequently, the difficulties that arise both in their learning and in their teaching, has multiple reasons. The truth is that there is a significant student aversion to mathematics, thus generating low levels of performance in this area of knowledge. In this article, two elements considered decisive for the learning of mathematics will be exposed. First, effective communication and language as the focus for teachers and students to reach agreements on the meanings of shared mathematical objects and, second, the affective domain, related to beliefs, attitudes and emotions, which they determine the subjective assessment that each individual shows when faced with learning processes related to mathematics and that they are precisely responsible for the reactions, more negative than positive, that they express.

KEY WORDS: Effective communication - Affective domain - Learning Mathematics.

\section{COMUNICAÇÃO EFICAZ E DOMINIO AFETIVO NO APRENDIZADO DE MATEMÁTICAS}

\section{RESUMO}

A matemática foi e seguirá sendo base rigorosa e inevitável em uma grande quantidade de disciplinas e especialidades, o que mostra a necessidade da sua inclusão e seu potencial em muitas propostas curriculares dentro da formação acadêmica em todos os níveis. Porém, diversos autores se referem a ela como a menos popular das disciplinas em quase qualquer plano de estudos. Esta falta de popularidade e, em consequência, as dificuldades que se apresentam tanto no aprendizado quanto no ensino, tem múltiplas razões. O certo é que está presente uma significativa aversão dos alunos para as matemáticas, gerando assim os baixos níveis de rendimento nesta área do conhecimento. No presente artigo, se irá expor dois elementos considerados determinantes para o aprendizado da matemática. No primeiro lugar, a comunicação efetiva e a linguagem como foco para que professores e alunos cheguem a acordos sobre os significados dos objetos matemáticos compartilhados e, em segundo lugar, o domínio afetivo, relacionado com as crenças, as atitudes e as emoções, que determinam a valorização subjetiva que cada indivíduo demonstra quando enfrenta processos de aprendizado relacionados com a matemática e que são, precisamente, os responsáveis das reações mais negativas que positivas, que expressam.

PALAVRAS CHAVE: Comunicação efetiva - domínio afetivo - aprendizado matemática. 
De la Oliva Fernández, M. Comunicación efectiva y dominio afectivo en el aprendizaje de las matemáticas

\section{Cómo citar el artículo:}

de la Oliva Fernández, M. (2020). Comunicación efectiva y dominio afectivo en el aprendizaje de las matemáticas. [Effective communication and affective domain in the learning of mathematics]. Revista de Comunicación de la SEECI, 53, 23-53. doi: http://doi.org/10.15198/seeci.2020.53.23-35

Recuperado de http://www.seeci.net/revista/index.php/seeci/article/view/662

\section{INTRODUCCIÓN}

Las iniciativas que se enfoquen en la mejora del proceso de aprendizaje de las matemáticas serán siempre oportunas debido, fundamentalmente, a la concepción de esta área disciplinar como ciencia compleja y por el ingrediente adicional de la naturaleza del que aprende, con la consiguiente valoración subjetiva que ese aprendiz demuestre.

En este contexto, se considera relevante la comprensión del pensamiento matemático y las consideraciones didácticas que de ella se derivan, entendiendo que la construcción de significados debe darse en ambientes de aprendizaje en los que, intencionadamente, se incorporen elementos complejos y contextualizados; donde exista la observación, la manipulación y la colaboración; en los que se permita al aprendiz la articulación de lo nuevo con lo ya conocido $y$, donde sea posible la reflexión. Ahora bien, ese intercambio productivo de aprendizaje descrito tendrá, como eje transversal, la comunicación efectiva como poderoso mecanismo de negociación de significados matemáticos y de desarrollo de estrategias de pensamiento, competencias fundamentales para seguir aprendiendo. En consecuencia, partiendo del hecho de que el mecanismo a través del cual compartimos ideas, problemas y opiniones es el lenguaje, debería ser obvio que las ideas, conceptos y problemas matemáticos también se transmiten a través de un lenguaje que llamamos técnico y cuyo desconocimiento suele ser causa de las mayores dificultades en el aprendizaje de la matemática.

Además de la complejidad intrínseca de la matemática, se debe concentrar la atención en la naturaleza del individuo que aprende. Para esto, se podrían delimitar los factores que determinan la valoración subjetiva que cada aprendiz demuestra cuando se enfrenta a la matemática o hacia procesos ligados a ella. Lo cierto es que estos factores están profundamente arraigados en los sujetos y son precisamente los responsables de las reacciones que tienen ante objetos de conocimiento matemático (Martínez, 2005). Entramos entonces en el ámbito del dominio afectivo, en el que se incluyen aspectos de distinta naturaleza que pueden ser sentimientos, emociones, creencias, actitudes, valores y apreciaciones (Gómez-Chacón, 2000).

\section{CONCEPCIÓN CONSTRUCTIVISTA DEL PENSAMIENTO MATEMÁTICO}

Uno de los retos centrales de la educación es la construcción, por parte del individuo, de significados contextualizados, culturalmente aceptados o socialmente válidos, por lo que una teoría constructivista global pasa a ser relevante. 
El constructivismo, concebido como una posición epistemológica, está basado en el papel activo del aprendiz y en la concepción del conocimiento como un proceso permanente de adaptación del sujeto a su entorno. Esto ha generado al menos tres posturas constructivistas básicas. La primera, llamada constructivismo simple o ingenuo, para aquellos que solo aceptan el primer supuesto; la segunda, constructivismo radical, para los que aceptan ambos supuestos $y$, una tercera postura, conocida como constructivismo social, para los que destacan la importancia del rol central que debe tener el conflicto cognitivo en la construcción del conocimiento (D'Amore, 2004).

En una concepción muy general del aprendizaje dentro del marco de la teoría constructivista, ante un nuevo objeto de conocimiento, cada individuo hace uso de sus esquemas y estructuras cognoscitivas, de sus percepciones y de sus conocimientos previos, para construir nuevos aprendizajes. En este proceso complejo, sus esquemas o estructuras se consolidan, sus percepciones se reformulan y sus conocimientos adquieren nuevos significados (Boggino, 1998). Sin embargo, esta es claramente una postura simple o ingenua, ya que no está presente el intercambio entre el aprendiz y quien medie ese aprendizaje.

Ahora bien, al concebir el conocimiento como un proceso permanente de adaptación del sujeto a su entorno, se asume la visión del constructivismo radical que, además, al incorporarse ese conflicto que surge del enfrentamiento entre nuestras concepciones previas sobre una determinada idea, objeto o concepto $y$, un nuevo estímulo que no encaja en el esquema que hemos construido o que se diferencia de otro con el que se tiene ocasión de compartir espacios de aprendizaje, estamos frente a la postura del constructivismo social. En definitiva, se considera el constructivismo de carácter global que incorpora al aprendiz en su rol protagónico, a su interacción con el entorno y a los conflictos cognitivos derivados de esa interacción.

Hasta aquí, la concepción expresada en el párrafo precedente, permite describir cómo ocurre el aprendizaje, pero no expone cómo debe ser el proceso de acompañamiento o de mediación por parte de quien figure como docente. Uno de los elementos a considerar en esa enriquecedora relación que debe establecerse entre alumno y profesor, es el hecho de que cada individuo tiene un estilo muy particular de aprender y que, esos estilos o modos, condicionan la forma de percibir el entorno, de procesar la información recibida y determinan la forma con la cual se comunican con el medio que los rodea.

Una vez entendido el fenómeno educativo integralmente, corresponde enfocar la atención en la concepción constructivista del pensamiento matemático. Para esto, es importante retomar de la psicología genética (Piaget en Boggino, 1998), la distinción de tres tipos de conocimiento: el físico, el social y el lógico-matemático y de resaltar la diferencia entre ellos. En particular, el conocimiento lógico-matemático puede ser concebido como una colección de relaciones mentales construidas por el sujeto en su interacción con los objetos de conocimiento, por lo que no existe por sí mismo en la realidad. Bajo esta perspectiva, depende de cada individuo construir las relaciones de 
seriación, clasificación, orden, inclusión, comparación, análisis, síntesis, reciprocidad y transitividad, entre otras, por lo cual el conocimiento no es el producto de la acción directa del aprendiz sobre los objetos, sino de la construcción que éste hace reflexivamente (Boggino, 1998). El conocimiento físico, por su parte, es el que se adquiere a través de la manipulación de los objetos que forman parte del entorno, de la realidad física y, se incorpora al sujeto, empíricamente. Por último, el conocimiento social es arbitrario y está directamente relacionado con las convenciones y consensos sociales y la fuente de este tipo de conocimiento, son los otros, con quienes compartimos espacios de aprendizaje.

A la luz de lo anteriormente expuesto, convendría que la didáctica de la matemática, se determinase por el tipo de conocimiento que le corresponde, es decir, el lógico-matemático. En este sentido, debido a la complejidad de cualquier noción matemática, donde podemos identificar elementos tales como el lenguaje (notaciones), las situaciones (problemas), las acciones (cómo resolver los problemas), los conceptos (definiciones), las proposiciones (propiedades) y los argumentos (demostraciones), se pone en evidencia la necesidad de un modelo didáctico que incorpore esta concepción constructivista del pensamiento matemático.

\section{COMUNICACIÓN EFECTIVA Y LENGUAJE EN EL APRENDIZAJE DE LA MATEMÁTICA}

El lenguaje concebido como la facultad humana que permite al individuo comunicarse consigo mismo y con el mundo exterior, implica el manejo de una serie de códigos, símbolos y sistemas de referencia organizados de acuerdo a determinadas leyes, con el propósito de expresar lo que el individuo experimenta en cada uno de los ámbitos de su vida.

A través del lenguaje, los seres humanos construyen su percepción del mundo, se relacionan con los demás, interpretan su entorno social, desarrollan potencial intelectual y, en definitiva, se incorporan en la época que les toque vivir.

Se podría afirmar que el hombre es producto del lenguaje, si se considera la concepción que expone Cadenas (2002), quien afirma que el lenguaje no sólo es el principal medio de comunicación del ser humano con el mundo, sino que, además, es el mecanismo a través del cual piensa y expresa sus sentimientos e ideas.

Ahora bien, si el lenguaje representa para el hombre el medio único y universal para su crecimiento y desarrollo, parece razonable suponer que debería ser considerado como el instrumento fundamental para el desarrollo de cualquier proceso de aprendizaje y de conocimiento de la realidad. Pero, además, en todas las actividades de carácter académico se hacen indispensables los procesos de comunicación tanto oral como escrita, fundamentando así, la clara relación entre el dominio de la lengua y el éxito en el desempeño del discente en su proceso de aprendizaje. Esta estrecha relación entre el lenguaje y otras áreas del conocimiento debe ser reforzada debido a que, por un lado, el lenguaje facilita la adquisición y transmisión de conocimientos y, por otro, las demás áreas del conocimiento aportan 
los contenidos sin los cuales, el desarrollo de la comunicación, se convierte en algo irrelevante y sin contexto (Cárdenas et al, 2001).

A lo largo de la evolución del conocimiento y, especialmente, en la de áreas específicas del saber, se han generado nuevos conocimientos, conceptos, definiciones y, en consecuencia, nuevas palabras, símbolos y formas de expresión. Así, por ejemplo, la matemática puede ser considerada un lenguaje universal, lo que implica que todos deberían manejar eficientemente las bases de este lenguaje. Como tal, la matemática ofrece unas reglas de uso general que son imprescindibles para el logro de aprendizajes significativos en esta área de conocimiento. Como se ha comentado en párrafos precedentes su aprendizaje ocasiona, en general, dificultades importantes a muchos debido, fundamentalmente, al hecho de tener que enfrentarse a un lenguaje diferente al cotidiano (o materno). Por esta razón, cabe poner la atención en la causa de estas dificultades considerando, además la enorme potencia de la matemática en la resolución de problemas y en la toma de decisiones.

Para Godino (et al, 2008), el desarrollo de un lenguaje para expresar ideas matemáticas, es esencial para comunicarse, toda vez que es el medio de comunicación con el entorno. En particular, dentro del contexto del aula, los docentes y discentes se valen del lenguaje como herramienta que les permite llegar a acuerdos sobre los significados de los objetos matemáticos que son compartidos.

Al reflexionar sobre la necesidad de comunicar lo descubierto, hacerlo conocido y transferible, queda en evidencia, lo indispensable de convenir, acordar y compartir un modo fácil de comunicación. Este modo de comunicación incluye lo que llamamos convenciones, que no son otra cosa que decisiones acordadas entre grupos de una misma lengua para entenderse en el uso, interpretación y significado de cada símbolo, palabra o elementos de lenguaje. Es decir, está implícito que nos referimos a un conocimiento social, que como mencionamos anteriormente, es arbitrario y está relacionado con consensos sociales, pero en este caso, entre personas del área específica. En este sentido, en el área de las matemáticas, se ha desarrollado y consolidado un lenguaje propio, a lo largo de los años: el lenguaje de las matemáticas.

Maier (1999), señala algunas características del lenguaje matemático, que lo diferencia del lenguaje cotidiano:

- En el lenguaje matemático no se aceptan elementos direccionales a menos que estén insertos en un sistema de referencia, previamente definido. Esto significa, por ejemplo, que las descripciones de representaciones geométricas en el espacio no aceptan términos como "arriba", "abajo", "a la izquierda", etc. Es necesario un sistema de coordenadas en el que no haya ambigüedades.

- Todos los conceptos matemáticos son ideales. Esto significa que, en una gran cantidad de estos conceptos, no hay ninguna relación con objetos reales, es decir, no se trata de conocimiento físico. También pueden definir relaciones ideales entre objetos o conjuntos de objetos (aunque éstos sean reales). Este 
carácter ideal de las matemáticas se traduce en que los conceptos deben ser construidos a través del discurso, cuya forma más completa es la definición.

- Esta idealización que se hace en matemática no permite ambigüedades, por lo tanto, cada objeto, término o símbolo está perfectamente definido, es único y cada significado particular corresponde a un solo objeto, término o símbolo.

- Las definiciones matemáticas son estrictas, lo que significa que deben diferenciarse claramente de términos similares del lenguaje cotidiano.

- El lenguaje matemático y, en consecuencia, su interpretación está limitada a un determinado ámbito propio de aplicación y siempre debe estar claramente definido.

Además de las características conceptuales mencionadas, también se encuentran dentro del lenguaje propio de las matemáticas, otros elementos que pueden ser organizados en dos grandes grupos: los elementos de organización y de representación de información, por un lado y, por otro, los elementos simbólicos. Con relación a los primeros, se puede encontrar una gran gama de herramientas para organizar y representar la información de la que se hace uso en matemáticas. Se habla entonces del uso de tablas, diagramas de Euler-Venn, diagramas circulares, diagramas de barras, sistemas de coordinadas, entre otros. En cuanto a los elementos simbólicos, nos referimos al uso frecuente en matemáticas de elementos más vinculados con el lenguaje cotidiano. Por ejemplo, debemos recordar que, en matemáticas, el uso de letras se constituye en una poderosísima herramienta para representar cantidades variables y realizar importantes generalizaciones.

Evidentemente, en este artículo no se pretende exhaustividad con todos los elementos específicos, símbolos y convenciones del lenguaje matemático, sino destacar la relevancia de conocerlo y posibilitar así la reflexión sobre la importancia que tiene para el logro de la comunicación efectiva en esta área de conocimiento e iniciar así procesos de aprendizaje más fluidos y significativos. Entonces el propósito de considerar el lenguaje cotidiano y matemático, de forma deliberada, es formar individuos con un manejo del intercambio comunicativo que les permita el respeto a las ideas ajenas, claridad y coherencia del mensaje, afectividad, adecuar el mensaje al contexto y aprender nuevos significados.

Tradicionalmente, la escritura y la comprensión de la lectura se considera una destreza que se "enseña" casi exclusivamente en el área de lengua y literatura, sin embargo, los docentes de todas las áreas esperan que el estudiante sea capaz de transferir este conocimiento a nuevos contextos, con otras características y esto, de entrada, no es cuestionable, lo que no debería ocurrir es que se abandone a los estudiantes en el desarrollo de las destrezas necesarias, yendo más allá de transmitir un cúmulo de conceptos sin asegurar su adecuada comprensión.

\section{EL DOMINIO AFECTIVO EN EL APRENDIZAJE DE LAS MATEMÁTICAS}

Un elemento de gran importancia en el aprendizaje de las matemáticas, más allá que lo que se ha expuesto hasta ahora, son los afectos (afectos, actitudes y creencias) sobre los individuos y su relación con el exitoso desempeño deseado. 
Los afectos resultan determinantes del contexto individual en el cual se vinculan los recursos, las estrategias y el autocontrol del que aprende (Gómez-Chacón, 2000). También influyen en la visión que cada aprendiz tiene sobre si mismo, alterando las relaciones que se producen con su propio sistema cognitivo. Igualmente, los afectos intervienen en el modo de organización social dentro de cualquier ambiente de aprendizaje $\mathrm{y}$, sin lugar a dudas, pueden constituirse en un obstáculo para el aprendizaje significativo. Es claro que la rigidez y la negatividad de las creencias que un alumno pueda tener acerca de la matemática y su aprendizaje, determinan las estrategias que utiliza, que, en muchos casos, se limitan a la memorización en lugar de intentar la comprensión.

La matemática ha estado presente en casi todas las actividades del hombre, sin lugar a dudas, por lo que resulta casi imposible encontrar algún fenómeno en el que se pueda evadir su magnífico poder explicativo. De hecho, la matemática ha sido fundamento riguroso de la gran mayoría de las disciplinas, mostrando su potencial en casi todas las propuestas curriculares dentro de la formación académica de todos los niveles. Sin embargo, como ya se ha mencionado, diversos autores la refieren como la más impopular de las disciplinas (Martínez, 2005).

La pobre popularidad que la matemática ha tenido tradicionalmente ha desfavorecido considerablemente tanto su aprendizaje como su enseñanza. En este sentido, algunos autores (Godino, 2008; Martínez, 2005; Gil et al, 2006) sustentan que la aversión de los estudiantes hacia la matemática apoya actitudes negativas hacia su aprendizaje. Esta es la razón por la cual el generalizado bajo rendimiento en esta área del conocimiento obliga a hacer una profunda reflexión de los factores afectivos y emocionales en el proceso de aprendizaje matemático.

Si analizamos la matemática como actividad intelectual, encontramos que su característica fundamental es la matematización, cuya definición apunta al proceso de construcción de modelos matemáticos. Esto se traduce en la secuenciación de procesos de organización y estructuración de la información que aparece en un problema, identificación de los aspectos matemáticos relevantes y el descubrimiento de regularidades, relaciones y estructuras (García, 2000). Dentro de este proceso pueden distinguirse dos etapas o momentos. Un primer momento en el cual se pasa desde el mundo real, al mundo de los símbolos (matematización horizontal), con los consecuentes procesos asociados de identificación, descubrimiento y transferencia y, un segundo momento, en el cual se tratan, matemáticamente, las situaciones e identificamos como procesos la representación, la utilización de modelos, la formulación, las pruebas y las generalizaciones (matematización vertical) (García, 2000).

Los procesos de matematización han definido, en función del apalancamiento de cada uno de los momentos (horizontal o vertical), distintas metodologías de enseñanza. Pero esta simplificación de la enseñanza de las matemáticas, es decir, asociándola exclusivamente a sus aspectos cognitivos, estaría evadiendo la complejidad de la matemática como disciplina $y$, en parte, limitando la compleja naturaleza del individuo que, en definitiva, es el que aprende. 
En cuanto a la complejidad de la matemática como disciplina, Andonegui (2004) afirma que es posible visualizarla desde diversas perspectivas. Destaca que pasa por aspectos epistemológicos asociados a cómo se construye cada objeto matemático, cómo se representa, cómo se relaciona con otros y cómo se valida el conocimiento matemático. Para este autor, otro elemento que abona a la complejidad de la matemática es el vinculado a la noción de contenidos que no son reales como la cantidad, la forma, el símbolo, la representación, la determinación y la incertidumbre, el cambio, entre otros. Por otra parte, el aspecto histórico constructivista permite el ejercicio de la imaginación, la intuición, la analogía, la metáfora, el análisis y la síntesis. Adicionalmente, se destacan dos aspectos que hacen de la matemática una ciencia compleja: la posibilidad del modelaje y aplicación a situaciones reales y, el aspecto estético de las simetrías, las regularidades, las generalizaciones y las singularidades.

Además de la dificultad intrínseca de la matemática, se debe poner especial atención a la complejidad de la naturaleza del que aprende. Para empezar, se podrían tratar de identificar los factores que determinan la valoración subjetiva que cada individuo demuestra cuando se enfrenta a la matemática o hacia procesos ligados a ella. Lo que sabemos al respecto es que, sin duda, esos factores están profundamente enraizados en los sujetos, lo que hace aún más difícil abordarlos desde el punto de vista de la didáctica de las matemáticas (Martínez, 2005).

Lo cierto es que, ese extenso abanico de sentimientos y estados de ánimo, que además pueden ser separados o considerados aparte del conocimiento, es lo que autores como Mcleod (1989, en Gil et al, 2006) definen como dominio afectivo, cuyos descriptores básicos pueden enfocarse en las creencias, actitudes y emociones (Gil et $a l, 2006)$.

Las creencias matemáticas son una componente del conocimiento del individuo en la experiencia y se definen como conocimientos y experiencias subjetivos del sujeto y están referidas tanto a los profesores como a los estudiantes. En ellas es posible distinguir dos elementos diferenciados, por una parte, la creencia de los estudiantes asociada a la matemática misma, en cuanto a su nivel de dificultad, a sus metodologías o a sus reglas, que evidentemente está más asociado al sistema educativo, al contexto escolar y a la actividad del aula. Pero, además, otro elemento puede ser identificado como la creencia del sujeto hacia su propia habilidad hacia la matemática (Gil et al, 2006).

La actitud hacia la matemática ha sido definida por Bazán (1997) como el fenómeno que involucra sentimientos (componente afectivo), creencias (componente cognitivo) y las tendencias de los sujetos a actuar de modo particular acercándose o alejándose del objeto "matemática". Para Bazán (1997) la actitud hacia la matemática puede verse desde las cuatro dimensiones que la conforman: la dimensión habilidad (confianza del sujeto en la propia habilidad matemática), a de aplicabilidad (valoración que hace el sujeto de la aplicación que puede tener la matemática), la de afectividad (asociada al agrado o desagrado hacia la matemática) y de ansiedad (las reacciones ansiosas hacia las matemáticas). 
De todas las definiciones revisadas sobre las actitudes, pueden inferirse una serie de aspectos importantes que, desde la perspectiva de Castro (2002) pasan por aceptar que las actitudes son adquiridas; suponen una alta carga afectiva y emocional; implican aceptación o rechazo del estímulo y se consideran valoraciones que traspasan las descripciones de esos estímulos; representan respuestas elegidas ante determinados valores; son entendidas como estructuras de múltiple dimensión; son experiencias subjetivas y constituyen aprendizajes estables por lo que pueden ser fomentadas, modificadas y aprendidas. Esta última característica se traduce en que es posible lograr cambios en la actitud hacia la matemática a través de elementos motivadores que permitan a los estudiantes realizar una posible reevaluación de sus actitudes hacia las matemáticas. Aquí es donde juega un papel tan importante conocer la potencia de las matemáticas como mecanismo modelador de situaciones reales, su alcance como desarrolladora de estrategias de pensamiento genérico, así como, su conexión o vinculación con la vida de los seres humanos, sus problemas y sus contextos.

Como tercer descriptor del dominio afectivo están las emociones, consideradas respuestas organizadas por encima de los sistemas psicológicos, que tienen una carga de significado positiva o negativa para el individuo. Por lo tanto, las emociones son el resultado complejo del aprendizaje, la influencia social y de la interpretación (Gómez-Chacón, 2000).

Gómez-Chacón (2003) señala otros aspectos relevantes en la dimensión afectiva del aprendizaje de la matemática. El primero de ellos vinculado a la comprensión del afecto como un sistema de representación en los individuos, es decir, que no puede ser considerado aislado o separado de la cognición, lo que significa que condiciona la apreciación cognitiva de los objetos de aprendizaje. El segundo aspecto está asociado al hecho de que los afectos tienen una base tanto biológica como social, porque el contexto social tiene una fuerte influencia sobre las creencias ya que se adquieren a través de procesos de transmisión cultural. El último aspecto que, esta experta en el tema de los afectos en el aprendizaje de la matemática, destaca es la distinción entre el afecto local y el global, indicando el alcance que ese afecto puede otorgarle a un individuo como parte de un determinado colectivo social.

Pero hay aún más, entendiendo el proceso de enseñanza aprendizaje como un todo complejo en el que son varios los actores que participan, conviene mencionar el dominio afectivo, pero desde el punto de vista del profesor. En este sentido, Gómez (1995) afirma que la calidad del profesor, está seriamente influida por su visión epistemológica con relación a los objetos de estudio matemáticos. El autor sugiere que esa visión puede estar ubicada en un continuo cuyos extremos están perfectamente diferenciados: la visión cerrada que es el paradigma a transmitir y la visión abierta de una actividad para la solución de problemas.

La visión epistemológica cerrada se afianza en la postura de ver el tema de estudio matemático como un conjunto de verdades y relega al estudiante a una postura totalmente pasiva como receptor de esas verdades (Gómez, 1995). Esto implica, para los estudiantes de profesores con esta visión epistemológica, el 
afianzamiento de creencias negativas hacia la matemática. Esto significa que el estudiante no puede crear espacios para distinguir los elementos de aprendizaje necesarios para la construcción de estructuras permanentes que le permitan aproximarse a nuevos temas y objetos matemáticos. Esta visión está totalmente centrada en los contenidos y no en los procesos de aprendizaje.

Por su parte, la visión epistemológica abierta en la cual los objetos de estudio matemático son concebidos como actividades en la búsqueda de las soluciones a problemas, permite que los estudiantes desarrollen las capacidades necesarias para utilizar creativamente el conocimiento, facilitando una visión más concreta y aplicada de los objetos matemáticos. De este modo, se fomenta la formación de creencias más constructivas acerca de la matemática (Gómez, 1995).

Queda en evidencia que los afectos y, con ellos, las creencias, las actitudes y las emociones, son factores fundamentales en la construcción de significados matemáticos $\mathrm{y}$, por tanto, en el desempeño de los aprendices, una propuesta didáctica en esta área no puede olvidar la consideración del aspecto afectivo en ella. Para esto, se deben tomar en cuenta elementos que van desde la comprensión y dominio de los contenidos matemáticos por parte del docente, como mecanismo para asegurar una visión, en los alumnos, no distorsionada, hasta la incorporación de elementos, en los curricula, relacionados con cognición y afecto, matemática como conocimiento cultural, así como el manejo del autoconcepto del alumno.

\section{CONCLUSIONES}

El aprendizaje de la matemática enfrenta importantes retos, toda vez que en cualquier noción matemática podemos identificar elementos tales como el lenguaje (notaciones), las situaciones (problemas), las acciones (cómo resolver los problemas), los conceptos (definiciones), las proposiciones (propiedades) y los argumentos (demostraciones), por lo que se pone en evidencia la necesidad de un modelo didáctico que incorpore esta concepción constructivista del pensamiento matemático.

Por su parte, el desarrollo y comprensión de un lenguaje para expresar las ideas matemáticas es esencial para comunicarse, toda vez que es el medio de intercambio con el entorno. En particular, dentro del contexto del aula, los docentes y discentes se valen del lenguaje como herramienta que les permite llegar a acuerdos sobre los significados de los objetos matemáticos que son compartidos. Por eso, al reflexionar sobre la necesidad de comunicar lo descubierto, hacerlo conocido y transferible, queda en evidencia, lo indispensable de convenir, acordar y compartir un modo fácil de comunicación efectiva.

Las creencias, las actitudes y las emociones tanto del docente como del discente, condicionan la forma en que ambos, a su manera y con sus particulares expectativas, abordan los objetos matemáticos en un espacio de aprendizaje, por lo que es fundamental la conciencia de este reto para indagar, en los estudiantes, sus particulares creencias y actitudes hacia los objetos de estudio matemáticos, con la 
De la Oliva Fernández, M. Comunicación efectiva y dominio afectivo en el aprendizaje de las matemáticas

certeza de que es posible que se modifiquen para lograr superar las barreras que tradicionalmente ha tenido el aprendizaje de la matemática.

\section{REFERENCIAS}

Andonegui, M. (2004). Interdisciplinariedad y educación matemática en la I y II etapa de la educación básica. Revista Iberoamericana de educación matemática, 8(26), 301-308. Recuperado de https://rieoei.org/historico/documentos/rie43a02.pdf

Bazán, J. (1997). Metodología estadística para la construcción de pruebas. Una aplicación al estudio de las actitudes hacia la matemática. (Tesis inédita de grado). UNALM. Lima.

Boggino, N. (1998). Psicogénesis de la matemática y articulación de niveles. Buenos Aires: Homo Sapiens Ediciones.

Cadenas, R. (2002). En torno al lenguaje. Caracas: Monte Ávila Editores.

Cárdenas, A., Castro, R. \& Soto, A. (2001). El desafío de la interdisciplinariedad en la formación docente. Revista electrónica Diálogos educativos, 1, 17-30. Recuperado de https://dialnet.unirioja.es/descarga/articulo/2095876.pdf

Castro, J. (2002). Análisis de los componentes actitudinales de los Docentes hacia la enseñanza de la Matemática. (Tesis inédita de doctorado). Universitat Rovira I Virgili, Tarragona.

D'Amore, B. (2004). El papel de la epistemología en la formación de profesores de matemática de la escuela secundaria. Epsilon: Revista de la Sociedad Andaluza de Educación Matemática "Thales"., 60. 413-434. ISSN 1131-9321.

García, J. (2000). La didáctica de las matemáticas. Una visión general. Recuperado de http://nti.educa.rcanaria.es/rtee/rtee.htm

Gil, N., Blanco, L. \& Guerrero, E. (2006). El dominio afectivo en el aprendizaje de las matemáticas. Electronic Journal of Research in Educational Psychology, 4 (1), 4772. doi : https://www.redalyc.org/articulo.oa?id=293123488003

Godino, J., Font, V. \& Wilhelmi, M. (2008). Análisis didáctico de procesos de estudio matemático basado en el enfoque ontosemiótico. Publicaciones, 38. 25-48. Recuperado de https://dialnet.unirioja.es/descarga/articulo/2763091.pdf

Gómez, P. (1995). Profesor: no entiendo. México: Grupo Editorial Iberoamérica.

Gómez-Chacón, I. (2000). Matemática emocional. Los afectos en el aprendizaje matemático. Madrid: Narcea, S.A. de Ediciones. 
De la Oliva Fernández, M. Comunicación efectiva y dominio afectivo en el aprendizaje de las matemáticas

Gómez-Chacón, I. (2003). La tarea intelectual en matemáticas, afectos, meta-afectos y sistemas de creencias. Boletín de la Asociación Matemática Venezolana, 10 (2), 225-247. Recuperado de https://www.emis.de/journals/BAMV/conten/vol10/igomez.pdf

Maier, R. (1999). El conflicto para los alumnos entre el lenguaje matemático y el lenguaje común. México: Grupo Editorial Iberoamérica.

Martínez Padrón, O. (2005). Dominio afectivo en educación matemática. Paradígma, 26 7-34.

Recuperado

de

http://ve.scielo.org/scielo.php?script=sci arttext\&pid=S1011-22512005000200002

\section{Autora:}

Mercedes de la Oliva Fernández.

Doctora en didáctica, organización escolar y didácticas especiales por la UNED, con amplia experiencia en el aprendizaje y la enseñanza de las matemáticas universitarias.

mdeoliva@gmail.com 